\title{
Preparation and exhaustive characterization of paraffin or palmitic acid microcapsules as novel phase change material
}

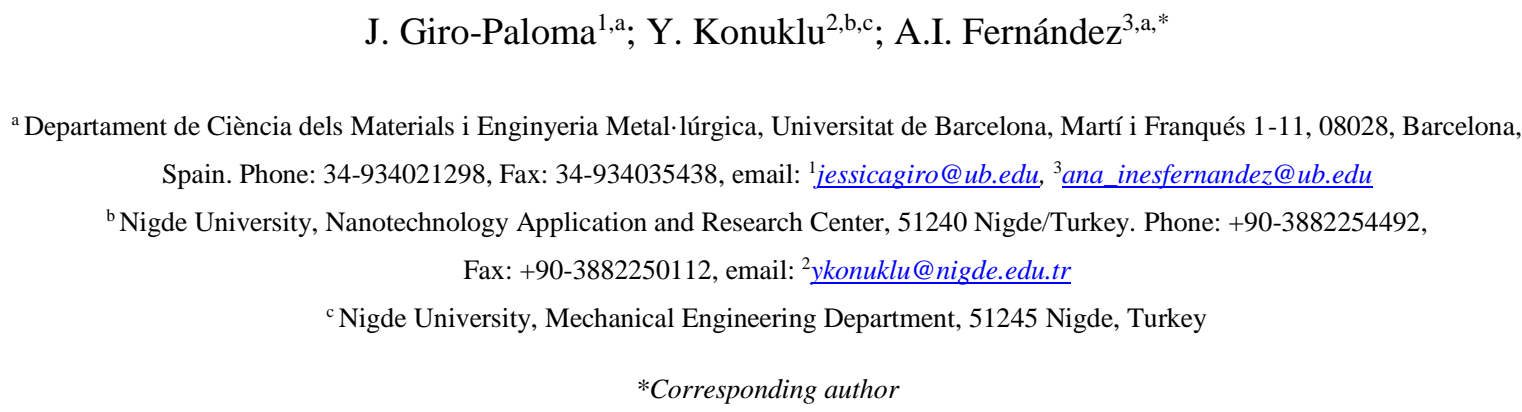

\section{Abstract}

In this study, two different types of Phase Change Materials (PCM) suitable for Thermal Energy Storage (TES) applications were used as a core material in a microencapsulation process. The wall material for these microencapsulated PCM (MPCM) was Poly(styrene-co-ethylacrylate) (PScEA). Microcapsules were prepared using an emulsion co-polymerization technique. The prepared MPCM were characterized as follows: morphology, shape and size were analyzed by Scanning Electron Microscopy (SEM) and Particle Size Distribution (PSD). Besides, Fourier Transformed Infrared spectroscopy (FT-IR) was used to perform the chemical characterization of the shell microcapsules. Moreover, thermophysical properties were analyzed by Differential Scanning Calorimetry (DSC) for the two PCM in usage (paraffin $42-44$ and palmitic acid) meanwhile the thermal stability was evaluated by Thermogravimetrical Analysis (TGA). Mechanical characterization of the prepared microcapsules was performed by using the Atomic Force Microscopy (AFM) as indentor. Experiments were performed at two different temperatures $25^{\circ} \mathrm{C}$ and $70{ }^{\circ} \mathrm{C}$, and two parameters were evaluated: the Young's modulus on a punctual area and the vertical force required to plastically deform the MPCM. At the light of the results, it can be considered that these synthesized MPCM were successfully prepared being able to be used in a TES system. 
Keywords: Phase Change Material, Microencapsulated Phase Change Material, Atomic Force Microscopy, Differential Scanning Calorimetry, Thermogravimetrical analysis, Fourier Transformed Infrared spectroscopy

\section{Introduction}

Phase Change Materials (PCM) are well known for Thermal Energy Storage (TES) applications and are reported as a promising energy technology for improving the energy efficiency (Gil, et al., 2010), hence different kind of PCM have been studied for different applications. To avoid the leakage of the PCM and control the variation in the storage material volume as the phase change takes place, the development of microencapsulated phase change materials (MPCM) has become an area of interest (Tyagi et al., 2011; Konuklu et al., 2014; Jamekhorshid et al., 2014; Zhang et al., 2004; Zhao and Zhang, 2011). MPCM are little containers made of polymeric shell and contains PCM inside (Alkan et al., 2009). There are three prerequisites for the microencapsulation process: the construction of the shell involving the PCM, ensuring that no leakage and no impurities are incorporated in the core/shell MPCM system. The second requirement is that the coating material thickness has to achieve the efficiency of the MPCM. By this way, MPCM third specification is being resistant to thermal and mechanical stresses. Besides, there are different methodologies to microencapsulate PCM (Boh and Šumiga, 2008; Jyothi et al., 2010; Sánchez et al., 2008). Based on the mechanisms of microcapsules formation, methodologies may be classified in chemical methods, physico-chemical methods, and physical and mechanical methods.

There are several possibilities or combinations of shell and core materials as a MPCM. Typical used shells are Poly(methyl methacrylate) (PMMA) (Alkan and Sar1, 2008; Wang et al., 2011) and melamine formaldehyde (MF) (Özonur et al., 2006), and the more usual PCM are paraffin (SánchezSilva et al., 2010) and particularly $n$-octadecane (Salunkhe and Shembekar, 2012; Zhang et al., 2012). Thus, there are a lot of recent studies with the preparation of MPCM made of PMMA and paraffin wax (Wang et al., 2012; Castellón et al., 2010; Ma et al., 2010), PMMA and n-octadecane (GiroPaloma, et al., 2013; Giro-Paloma, et al., 2014), MF and paraffin wax (Su et al., 2012; Šumiga et al., 2011), and finally MF and $n$-octadecane (Zhang and Wang, 2009). Although these two polymers are the most popular ones due their characteristics, polystyrene (PS) is also a popular container used as a shell in MPCM manufacturing. It can be mixed with different type of PCM, depending on the application and in the comfort temperature. Borreguero et al. (2011) produced by suspension polymerization technique microcapsules containing RT-27 and PS as a shell material. Although the MPCM was obtained, they concluded that for this kind of PCM is much better a shell of PE-EVA (polyethylene - ethyl vinyl acetate) because its thermal stability is better. Moreover, Sánchez et al. (2010) synthesized by suspension like polymerization MPCM for textiles made PS as a shell and paraffin wax as a core. Other authors used the same polymeric shell and changed the PCM obtaining 
MCPMs to be used for Phase Change Slurries (PCS) and phase change emulsions, as Yang et al. (2003), where they prepared and characterized microcapsules containing $n$-tetradecane for improving the heat transfer ability and energy transport ability. Besides, Fang et al. (2008) studied nanoencapsulated PCM with PS $n$-octadecane, as a shell and as PCM, respectively. Their conclusion was that these capsules were successfully prepared by the ultrasonic assistant miniemulsion in-situ polymerization. Also, Sánchez-Silva et al. (2010) studied the MPCM of styrene mixed with another polymer in paraffin. The preparation of the microcapsules was done by suspension - like polymerization, and the main conclusion for the styrene-methyl methacrylate with paraffin was that it is impossible to microencapsulate the paraffin wax when the MMA/St is equal to 2.0. Also, styrene can be copolymerized with other substances creating the ABS (Acrylonitrile-Styrene-Butadiene), AB (Acrylonitrile-styrene copolymer), and the SBS (Styrene-butadiene-styrene). Examples of these are the ones of Yang et al. (2009) and Kenisarin and Kenisarina (2012), respectively. The first one prepared MPCM of ABS and AS with $n$-tetradecane in the core, remarking that their potential and flexibility as a shell material. The SBS MPCM study includes microparticles with paraffin wax as a core material and others with lauric acid.

In our literature survey, none reported preparation of paraffin and palmitic acid microcapsules with Poly(styrene-co-ethylacrylate) (PScEA) shells. In this paper is reported the microencapsulation and full characterization of paraffin and palmitic acid in PScEA shells by the emulsion copolymerization method to obtain micro-nanocapsules for low temperatures solar thermal applications by Scanning Electron Microscopy (SEM), Particle Size Distribution (PSD), Fourier Transformed Infrared Spectroscopy (FT-IR), Differential Scanning Calorimeter (DSC), Thermogravimetrical Analysis (TGA), Atomic Force Microscopy (AFM).

\section{Materials and methods}

\subsection{Materials}

Paraffin 42 - 44 and palmitic acid were used as core PCM. Styrene (> $99 \%$; Sigma Aldrich Company, USA) and ethyl acrylate, $\mathrm{C}_{5} \mathrm{H}_{8} \mathrm{O}_{2}(>99 \%$; Merck, Germany) were used as a shell material; ethylene glycol dimethacrylate (EGDMA), $\mathrm{C}_{10} \mathrm{H}_{14} \mathrm{O}_{4}$ (Merck, Germany) was used as a crosslinking agent and it was distilled before use. The initiator of ammonium peroxodisulfate (Merck, Germany) and other analytical reagents tert-butylhydroperoxide (70 \%; Merck, Germany), Triton X-100 (Merck, Germany), iron (II) sulfate 7-hydrate $\left(\mathrm{FeSO}_{4} \cdot 7 \mathrm{H}_{2} \mathrm{O}\right)$ (Panreac, Spain), and sodium thiosulfate $\left(\mathrm{Na}_{2} \mathrm{~S}_{2} \mathrm{O}_{3}\right)$ (Merck, Germany) were used without further purification. 


\subsection{Preparation of microcapsules}

A typical emulsion polymerization process technique was used in the preparation of MPCMs with PScEA shell. The emulsion co-polymerization method consists on mixing the polymer in an oiled system, adding an emulsifier. An emulsification is needed to create a water/oil emulsion and to generate a crosslinked system. Then, it will be needed the wash of the emulsion, to eliminate the oil, creating isolated microcapsules. Firstly, $120 \mathrm{ml}$ of deionized water, $1.60 \mathrm{~g}$ of Triton X100, $28 \mathrm{~g}$ of core material were stirred for 30 minutes at $40{ }^{\circ} \mathrm{C}$. Then $14 \mathrm{~g}$ of styrene, $14 \mathrm{~g}$ of ethyl acrylate, $8 \mathrm{~g}$ of EGDMA, $1 \mathrm{ml}$ of $\mathrm{FeSO}_{4} \cdot 7 \mathrm{H}_{2} \mathrm{O}$ solution and $0.25 \mathrm{~g}$ of ammonium peroxodisulfate was stirred in a beaker, then added to emulsion and stirring was continued at $1000 \mathrm{rpm}$. Then $0.25 \mathrm{~g}$ of $\mathrm{Na}_{2} \mathrm{O}_{3} \mathrm{~S}_{2}, 1 \mathrm{ml}$ of tert-butylhydroperoxide was added to the emulsion and heated to $95{ }^{\circ} \mathrm{C}$. The solution was stirred for 5 hours and then it was cooled down to room temperature and washed with water five times. Finally, the microcapsules were rinsed with deionized water, filtered and dried approximately 72 hours at room conditions.

\subsection{Characterization of microcapsules}

\subsubsection{Scanning Electron Microscopy (SEM)}

The morphology of the samples was characterized using an environmental Scanning Electron Microscopy (Zeiss Evo 50, Zeiss, UK). The samples were fixed on the sample holder using a doublesided tape and then the particle size and the morphology of the samples were observed. The conditions were low vacuum and high voltage $(15 \mathrm{kV})$, obtaining the image by secondary electrons.

\subsubsection{Particle Size Distribution}

The average particle size and size distribution of the micro-nanoPCMs were determined using a Nano Zetasizer (Malvern Instruments Ltd., UK). Measurements were carried out at $25{ }^{\circ} \mathrm{C}$. To avoid multiple scattering effects, firstly, MPCM was mixed with water (1:100) and stirred 30 minutes in ultrasonic bath. The mean particles diameters were calculated from the average of three measurements.

\subsubsection{Fourier-Transformed Infrared Spectroscopy (FT-IR)}

FT-IR spectroscopy is a potent technique to identify functional groups in organic polymers or compounds. The chemical characterization of the MPCM shells was performed by a Fourier 
Transformed Infrared (FT-IR) spectroscopy. It was used through FT-IR Spectrum Two ${ }^{\mathrm{TM}}$ from Perkin Elmer using a working range from 400 to $4000 \mathrm{~cm}^{-1}$.

(1)

\subsubsection{Differential Scanning Calorimeter (DSC)}

The thermal properties melting and solidification temperature and the melting/solidification enthalpy of the two types of microcapsules containing PCM were evaluated with a DSC (Perkin Elmer Diamond) with a heating-cooling rate of $5^{\circ} \mathrm{C} \cdot \mathrm{min}^{-1}$ in the range of $10-80{ }^{\circ} \mathrm{C}$. During DSC analysis, about $5 \mathrm{mg}$ of sample was used. This technique shows the melting temperature $\left(\mathrm{T}_{\mathrm{m}}\right)$ and solidification temperature $\left(\mathrm{T}_{\mathrm{s}}\right)$ of the sample and the enthalpy value for each process (melting and solidification, $\mathrm{H}_{\mathrm{m}}$ and $\mathrm{H}_{\mathrm{s}}$ respectively) which is equivalent to the area under the curve. The determination of the phase change material content in the MPCMs followed from the formula:

$$
\boldsymbol{P C M}(\%)=\Delta \boldsymbol{H}_{\text {microPCM }} / \Delta \boldsymbol{H}_{\text {corePCM }} \cdot \mathbf{1 0 0} \quad \text { Equation } 1
$$

\subsubsection{Thermogravimetrical Analysis (TGA)}

The thermal stability of the microcapsules was evaluated with a Simultaneous SDTQ600 TA Instruments under $\mathrm{N}_{2}$ and air atmosphere. The scanning rate was $10{ }^{\circ} \mathrm{C} \cdot \mathrm{min}^{-1}$ in the temperature range between 25 and $600{ }^{\circ} \mathrm{C}$ for $5.4420 \mathrm{mg}$ of sample for both samples.

\subsubsection{Atomic Force Microscopy (AFM)}

Samples were suspended in ethanol, and $50 \mu \mathrm{l}$ of suspension was poured on freshly cleaved mica and gently dried with a nitrogen stream. Then, sample was imaged in air. The experiments were performed in air using a Peak Force Quantitative NanoMechanics mode (QNM) with a system Multimode 8, Nanoscope V electronics. The AFM probe was a diamond MDNISP one, with a nominal spring constant: $388 \mathrm{nN} \cdot \mathrm{nm}^{-1}$. The sensitivity was $68.2 \mathrm{~nm} \cdot \mathrm{V}^{-1}$ with a set point between 400 and $550 \mathrm{nN}$. Moreover, the working conditions for the peak force amplitude were $150 \mathrm{~nm}$, and the DMT Modulus limit 1 TPa. Both samples A and B were more soluble in ethanol than water. In order to break the aggregates, the samples were sonicated until the solution was homogeneous. Capsules $\mathrm{A}$ was tested at $25^{\circ} \mathrm{C}$ and $50{ }^{\circ} \mathrm{C}$, and Capsules $\mathrm{B}$ at $25^{\circ} \mathrm{C}$ and $70{ }^{\circ} \mathrm{C}$ to evaluate the different behavior of the MPCM when the PCM is solid (around $25^{\circ} \mathrm{C}$ ) and liquid (up to $43^{\circ} \mathrm{C}$ for A and up to $63{ }^{\circ} \mathrm{C}$ for B). It was measured the maximum force that is needed to apply at the top of a capsule to break it, as well as a Young's modulus map and Young's modulus histogram for some capsules at different temperatures. 
Individual indentation tests were made in diverse particles of the upper flat-top part of the capsules in order to avoid tip slippage due to sample curvature. For all the force vs. Z graph representations, the blue line corresponds to tip sample approach process and the red one corresponds to the tip retraction from the sample. The first region corresponds to the no contact between the tip and the sample. Then, when the tip touches the surface of the sample, the cantilever applies an increasing force on the sample, deforming it elastically. Finally, the retraction of the AFM probe is represented by the red line. It is essential to notice that a three-sided dent in the upper site of the particle can be observed when it is plastically deformed by the AFM probe. Also, it has to be remarkable that usually the general profile of the particles seem to be triangular due to the pyramidal form of the AFM tip, and not due to the real profile of the particle. To calculate the force F, the following equation is used:

$$
\mathrm{F}=\mathrm{k} \cdot \Delta_{\mathrm{z}}
$$

Equation 2

where $k$ is the AFM probe, and $\Delta_{\mathrm{z}}$ is cantilever deflection in the $\mathrm{z}$ axis. Besides, the cantilever deflection is expressed in Equation 3 as:

$$
\Delta_{z}=\Delta V / S
$$

Equation 3

where $\Delta \mathrm{V}$ is the increment in photodetector vertical signal as the tip contacts the sample. The $\mathrm{S}$ value is the sensitivity, which is the slope of the contact region of a force curve performed on a rigid sample. It is also needed to take into account the sample penetration $(\delta)$ because of the applied force value:

$$
\delta=z-\Delta_{z}
$$

Equation 4 where $z$ represents the piezo-scanner displacement in the axis perpendicular to the sample plane. The F vs. $z$ graphs achieved at a certain force value, and it was evaluated by the Hertz model in the elastic region with Equation 5:

$$
F=\left(3 / 4 \cdot E_{\text {eff }} \cdot R^{1 / 2}\right) \cdot \delta^{3 / 2}
$$

Equation 5

Besides, the Young Modulus is considered following Equation 6:

$$
1 / E_{\text {eff }}=\left[\left(1-v^{2}\right) / E\right]+\left[\left(1-v_{i}^{2}\right) / E_{i}\right]
$$
and Young Modulus ( $\left.\mathrm{E}_{\mathrm{eff}}\right)$ value can be obtained using Equation 6, taking into consideration that $v$ is the Poisson ratio with 0.33 as a value. Furthermore, the subindex $i$ corresponds to the mechanical properties of the AFM probe, $\mathrm{SiO}_{2}$. The values for the $\mathrm{E}_{\mathrm{i}}$ and $v_{\mathrm{i}}$ are $76 \mathrm{GPa}$ (Namazu and Isono, 2003) and 0.17 respectively (Oliver and Pharr, 1992). 


\section{Results and discussion}

\subsection{Scanning Electron Microscopy (SEM)}

Figure 1 shows SEM micrographs of the PScEA shell microcapsules obtained using emulsion copolymerization technique. As it can be seen, microparticles almost have a spherical structure and the microcapsule stability after the coating process was confirmed.

\section{Figure 1.}

\subsection{Particle Size and Distribution Analysis (PSD)}

Particle sizes distributions of sample A and sample B are shown in Figure 2. Mean particle diameters for sample A and sample B were 165 and $265 \mathrm{~nm}$, respectively. Smaller and uniform microcapsules were obtained with PScEA + paraffin.

Figure 2.

\subsection{Fourier-Transformed Infrared Spectroscopy}

The FTIR spectra of core material, shell material and MPCM are shown in Figure 3 and Figure 4. Each of the characteristic peaks belonging to the core material preserves itself after microencapsulation. These results in Transmittance (\%) vs. Wavenumber $\left(\mathrm{cm}^{-1}\right)$ confirm that the encapsulation of paraffin and palmitic acid could be prepared within PScEA.

Figure 3.

Figure 4.

In general, FT-IR spectroscopy is used to describe the microcapsules shell structure. In this work, FT-IR shell spectra of the two samples under study were given in Figure 5 to verify the presence of PScEA in both MPCM. 
Figure 5.

\subsection{Differential Scanning Calorimeter (DSC)}

Figure 6 provides the curves of the DSC samples containing 32.1 wt.\% paraffin and 47.8 wt.\% palmitic acid at $5{ }^{\circ} \mathrm{C} \cdot \mathrm{min}^{-1}$ scanning rate. Moreover, in Table 1 is shown the DSC numerical results for capsule A PScEA with Paraffin 42 - 44 in the core, and capsule B PScEA with palmitic acid as the PCM. It is indicated the onset melting temperature $\left(\mathrm{T}_{\mathrm{om}}\right)$ of the DSC curve, the melting peak temperature $\left(\mathrm{T}_{\mathrm{pm}}\right)$, the endset melting tempertaure $\left(\mathrm{T}_{\mathrm{em}}\right)$, the melting enthalpy $\left(\mathrm{H}_{\mathrm{m}}\right)$, onset the crystallazing temperature $\left(\mathrm{T}_{\mathrm{oc}}\right)$, the cristallazing peak temperature $\left(\mathrm{T}_{\mathrm{pc}}\right)$, the endset cristallizing temperature $\left(\mathrm{T}_{\mathrm{ec}}\right)$, and finally the cristallization enthaply $\left(\mathrm{H}_{\mathrm{c}}\right)$. Figure $6 \mathrm{a}$ and Table $\mathbf{1}$ indicate that the peak temperatures of melting and freezing were determined to be $44.23{ }^{\circ} \mathrm{C}$ and $36.84{ }^{\circ} \mathrm{C}$ for nonencapsulated A and $42.39^{\circ} \mathrm{C}$ and $37.41^{\circ} \mathrm{C}$ for capsule A. Furthermore, Figure $6 \mathrm{~b}$ and Table 1 indicate that the peak temperatures of melting and freezing were determined to be $64.57^{\circ} \mathrm{C}$ and $57.00{ }^{\circ} \mathrm{C}$ for non-encapsulated $\mathrm{B}$ and $62.66{ }^{\circ} \mathrm{C}$ and $56.22{ }^{\circ} \mathrm{C}$ for capsule $\mathrm{B}$. It is clear that, there is no significant temperature change observed after microencapsulation process. It was determined that, the microcapsules obtained in this study, capsule A and capsule B, are very suitable for thermal energy storage applications. Moreover, the enthalpy values are $49 \mathrm{~J} \cdot \mathrm{g}^{-1}$ for capsule A and $97 \mathrm{~J} \cdot \mathrm{g}^{-1}$ for capsule $\mathrm{B}$, which are very good values according to the reported data for the studied temperature ranges, thus being a good alternative for solar thermal applications.

Figure 6.

Table 1.

\subsection{Thermogravimetrical Analysis (TGA)}

Thermal stabilities of the MPCM samples were evaluated by the TGA analysis. The two type of capsules showed two-step decomposition profiles (Figure 7). The first-step weight loss of the microcapsules is due to the degradation of the core materials (paraffin wax and palmitic acid) and the second-step decomposition results from the degradation of the PScEA copolymer shell. Over $470{ }^{\circ} \mathrm{C}$ the microcapsules decomposed completely. For capsule A the first weight loss corresponds to the 
decomposition of the PCM paraffin wax around $200{ }^{\circ} \mathrm{C}$ and the associated loss is around a $40 \%$ of the sample. The second mass loss involves approximatelly the $60 \%$ of the sample mass with an onset temperature around $275^{\circ} \mathrm{C}$, being completely decomposed at $430{ }^{\circ} \mathrm{C}$. Moreover, it can be observed the thermal degradation for the capsule $\mathrm{B}$, where the first weight loss is due to the palmitic acid decomposition, representing almost the $50 \%$ by weight, and the second one fits with the shell, being completeley decomposed at $450{ }^{\circ} \mathrm{C}$. From these results, capsule B with higher melting point is more stable than capsule A.

Figure 7.

\subsection{Atomic Force Microscopy (AFM)}

Considering one isolated capsule, and selecting a specific delimited area, it can be studied a Young's modulus map and the histogram of the sample A at $25^{\circ} \mathrm{C}$, as Figure 8 shows. The mean Young's modulus value for this specific region is $106 \mathrm{MPa}$.

$$
\text { Figure 8. }
$$

In Figure 9 is shown the vertical force $(\mu \mathrm{N})$ vs. sample penetration $(\mathrm{nm})$ curve obtained by processing the measurement of the cantilever deflection at $25{ }^{\circ} \mathrm{C}$. In view of all the equations and considerations explained previously, the average breakthrough force after three repetitions for sample A at $25^{\circ} \mathrm{C}$ is $3.9 \mu \mathrm{N}$. It can be noticed in b) point that there is a slight change in the slope curve, due to the breakage of the microcapsule shell.

Figure 9.

It is needed to increase the temperature up to $50^{\circ} \mathrm{C}$ to observe the mechanical differences. Around this value is reached the phase change temperature, so the PCM will be in liquid state.

It is seen in Figure 10 that the average Young's modulus histogram for the specific area delimited in the left image is $160 \mathrm{MPa}$. For this reason, it can be concluded that for sample A the Young's 
modulus value looks independent from temperature, because the values obtained at room temperature and at $50{ }^{\circ} \mathrm{C}$ are extremely small.

Figure 10.

Besides, in Figure 11 is shown that the sample A at $50{ }^{\circ} \mathrm{C}$ has a maximum force before breaking at $5.4 \mu \mathrm{N}$, which is almost the same than at room temperature.

\section{Figure 11.}

For sample A it can be concluded that the differences in temperature are not very significant, because the results at both temperatures $\left(25^{\circ} \mathrm{C}\right.$ and $\left.50{ }^{\circ} \mathrm{C}\right)$ are quite similar for the Young's modulus value and also for the maximum force that can be applied on the top of the microcapsules before breaking them. For this reason, it can be concluded that this sample A is suitable for using it at both temperatures without suffering any mechanical deformation. Otherwise, when sample B is studied, we can observe some more evidence differences.

Then, in Figure 12 is evaluated the Young's modulus in the specific area delimited with the black frame at $25^{\circ} \mathrm{C}$ for sample B. The result gives a mean value of $422 \mathrm{MPa}$.

\section{Figure 12.}

The typical graph of loading-unloading is shown in Figure 13 and denotes that for capsule B at room temperature there is a breakthrough force of $17.3 \mu \mathrm{N}$.

\section{Figure 13.}

For capsule $\mathrm{B}$ case, it was decided to increase the temperature until $70^{\circ} \mathrm{C}$ due to the phase change temperature of the PCM. 
These microcapsules gives a result of $517 \mathrm{MPa}$ at $70{ }^{\circ} \mathrm{C}$ when it is evaluated its Young's modulus, as Figure 14 shows. Young's modulus values for room temperature and $70^{\circ} \mathrm{C}$ are low, for this reason, the difference between them is not representative; hence, it can be assumed that $E$ values do not change in front of the temperature.

Figure 14.

Besides, in Figure 15 it is shown the result for the maximum allowed force on the top of the capsule B at $70{ }^{\circ} \mathrm{C}$. This value is around the $1.8 \mu \mathrm{N}$, ten times lower compared with $25^{\circ} \mathrm{C}$ due to the experimental temperature. This result is really important because this sample can be damaged in an easiest way than the capsule A at higher temperatures.

Figure 15.

\section{Conclusions}

In this research, paraffin and palmitic acid were microencapsulated with a Poly(stirene-coethylacrylate) PScEA shell via an emulsion co-polymerization technique. SEM results confirm that paraffin (capsule A) and palmitic acid (capsule B) encapsulated successfully in this study, and the diameters for capsule A and capsule B were analyzed by PSD being $166 \mathrm{~nm}$ and $265 \mathrm{~nm}$, respectively. Besides, the encapsulated paraffin and palmitic acid can be considered to have good potential for energy storage. Also, TGA results denoted a slightly higher thermal stability for sample B in the same working conditions. The FTIR results proved that paraffin and palmitic acid encapsulated successfully with a PScEA shell in this study. AFM results confirm that there are no differences in capsule A for Young's modulus and force applied to break the microcapsule at different temperatures $\left(25^{\circ} \mathrm{C}\right.$ and 50 ${ }^{\circ} \mathrm{C}$ ) because the results are similar. Capsule B does not have the same mechanical behavior at $25{ }^{\circ} \mathrm{C}$ and $70{ }^{\circ} \mathrm{C}$, the force is ten times lower at $70{ }^{\circ} \mathrm{C}$ comparing the results with those at $25^{\circ} \mathrm{C}$ although the Young's modulus has a similar value.

\section{Acknowledgment}


The work is partially funded by the Spanish government (ENE2011-28269-C03-02). The authors would like to thank the Catalan Government for the quality accreditation given to their research group DIOPMA (2014 SGR 1543). The research leading to these results has received funding from the European Union's Seventh Framework Programme (FP7/2007-2013) under grant agreement $n^{\mathbf{o}}$ PIRSES-GA-2013-610692 (INNOSTORAGE). Also, preparation of microcapsules funded by The Scientific \& Technical Research Council of Turkey (TUBITAK) (The Project Code: TUBITAK 111M614).

\section{References}

Alkan, C., Sar1, A., 2008. Fatty acid/poly(methyl methacrylate) (PMMA) blends as form-stable phase change materials for latent heat thermal energy storage. Sol. Energ. 82, 118-124.

Alkan, C., Sarı, A., Karaipekli, A., Uzun, O., 2009. Preparation, characterization, and thermal properties of microencapsulated phase change material for thermal energy storage. Sol. Energ. Mat. Sol. C.;93:143-7.

Boh, B., Šumiga, B.,2008. Microencapsulation technology and its applications in building construction materials. RMZ - Materials and Geoenvironment 55(3):329-44.

Borreguero, A.M., Valverde, J.L., Rodríguez, J.F., Barber, A.H., Cubillo, J.J., Carmona, M., 2011. Synthesis and characterization of microcapsules containing Rubitherm ${ }^{\circledR}$ RT27 obtained by spray drying. Chem. Eng. J. 166, 384-390.

Castellón, C., Medrano, M., Roca, J., Cabeza, L.F., Navarro, M.E., Fernández, A.I., Lázaro, A., Zalba, B., 2010. Effect of microencapsulated phase change material in sandwich panels. Renew. Energ. 35, $2370-2374$.

Fang, Y., Kuang, S., Gao, X., Zhang, Z., 2008. Preparation and characterization of novel nanoencapsulated phase change materials. Energ. Convers. Manage. 49, 3704-3707.

Gil, A., Medrano, M., Martorell, I., Lázaro, A., Dolado, P., Zalba, B., Cabeza, L.F., 2010. State of the art on high temperature thermal energy storage for power generation. Part 1 - concepts, materials and modellization. Renew. Sust. Energ. Rev. 14, 31-55.

Giro-Paloma, J., Oncins, G., Barreneche, C., Martínez, M., Fernández, A.I., Cabeza, L.F., 2013. Physico-chemical and mechanical properties of microencapsulated phase change material. Appl. Energ. 109, 441-448. 
367 Giro-Paloma, J., Barreneche, C., Delgado, M., Martínez, M., Fernández, A.I., Cabeza, L.F., 2014.

368 Physicochemical and thermal study of a MPCM of PMMA shell and paraffin wax as a core. Energy 369 Procedia 48, 347-354.

370 Jamekhorshid, A., Sadrameli, S.M., Farid, M., 2014. A review of microencapsulation methods of 371 phase change materials (PCMs) as a thermal energy storage (TES) medium. Renew. Sust. Energ. Rev. $372 \quad 31,531-542$.

373 Jyothi, N., Prasanna, M., Prabha, S., Ramaiah, P.S., Srawan, G., Sakarkar, S., 2010. 374 Microencapsulation Techniques, Factors Influencing Encapsulation Efficiency. J. Microencapsul. $375 \quad 3187-97$.

376 Kenisarin, M.M., Kenisarina, K.M., 2012. Form-stable phase change materials for thermal energy 377 storage. Renew. Sust. Energ. Rev. 16, 1999-2040.

378 Konuklu, Y., Unal, M., Paksoy, H.Ö., 2014. Microencapsulation of caprylic acid with different wall 379 materials as phase change material for thermal energy storage. Sol. Energ. Mat. Sol. C. 120B, 536542.

Ma, S., Song, G., Li, W., Fan, P., Tang, G., 2010. UV irradiation-initiated MMA polymerization to prepare microcapsules containing phase change paraffin. Sol. Energ. Mat. Sol. C. 94, 1643-1647.

Namazu, T., Isono, Y., 2003. Quasi-satic bending test of nano-scale $\mathrm{SiO}_{2}$ wire at intermediate temperatures using AFM-based technique. Sensor. Actuat. A-phys. 194, 78-85.

Oliver, W.C., Pharr, G.M., 1992. An improved technique for determining hardness and elastic modulus using load and displacement sensing indentation experiments. J. Mater. Res. 7(6), 15641583.

Özonur, Y., Mazman, M., Paksoy, H.Ö., Evliya, H., 2006. Microencapsulation of coco fatty acid mixture for thermal energy storage with phase change material. International Journal Energy Research 30 (10), 741-749.

Salunkhe, P.B., Shembekar, P.S., 2012. A review on effect of phase change material encapsulation on the thermal performance of a system. Renew. Sust. Energ. Rev. 16, 5603-5616. conditions on the microencapsulation of PCMs by means of suspension-like polymerization. Colloid. Polym. Sci. 286:1019-27. 
397 Development of thermo-regulating textiles using paraffin wax microcapsules. Thermochim. Acta. 498, 16-21.

Sánchez-Silva, L., Rodríguez, J.F., Romero, A., Borreguero, A.M., Carmona, M., Sánchez, P., 2010. Microencapsulation of PCMs with a styrene-methyl methacrylate copolymer shell by suspension-like polymerization. Chem. Eng. J. 157, 216-222.

Su, J.F., Wang, X.Y., Dong, H., 2012. Influence of temperature on the deformation behaviours of melamine-formaldehyde microcapsules containing phase change material. Mater. Lett. 84, 158-161.

404

Šumiga, B., Knez, E., Vrtacnik, M., Ferk-Savec, V., Staresinic, M., Boh, B., 2011. Production of Melamine-Formaldehyde PCM Microcapsules with Ammonia Scavenger used for Residual Formaldehyde Reduction. Acta. Chim. Slov. 58, 14-25.

Tyagi, V.V., Kaushik, S.C., Tyagi, S.K., Akiyama, T., 2011. Development of phase change materials based microencapsulated technology for buildings: A review. Renew. Sust. Energ. Rev. 15(2), 13731391.

Wang, Y., Dong Xia, T., Xia Feng, H., Zhang, H., 2011. Stearic acid/polymethylmethacrylate composite as form-stable phase change materials for latent heat thermal energy storage. Renew. Energ. 36, 1814-1820.

Wang, Y., Shi, H., Xia, T.D., Zhang, T., Feng, H.X., 2012. Fabrication and performances of microencapsulated paraffin composites with polymethylmethacrylate shell based on ultraviolet irradiation-initiated. Mater. Chem. Phys. 135, 181-187.

Yang, R., Xu, H., Zhang, Y., 2003. Preparation, physical property and thermal physical property of phase change microcapsule slurry and phase change emulsion. Sol. Energ. Mat. Sol. C. 80, 405-416.

Yang, R., Zhang, Y., Wang, X., Zhang, Y., Zhang, Q., 2009. Preparation of n-tetradecane-containing microcapsules with different shell materials by phase separation method. Sol. Energ. Mat. Sol. C. 93, $1817-1822$.

Zhang, G.H., Bon, S.A.F, Zhao, C.Y., 2012. Synthesis, characterization and thermal properties of novel nanoencapsulated phase change materials for thermal energy storage. Sol. Energ. 86, 11491154.

Zhang, H., Wang, X., 2009. Fabrication and performances of microencapsulated phase change materials based on n-octadecane core and resorcinol-modified melamine-formaldehyde shell. Colloid. Surface. A. 332, 129-138. 
427 Zhang, X.X., Tao, X.M., Yick, K.L., Wang, X.C., 2004. Structure and thermal stability of 428 microencapsulated phase-change materials. Colloid. Polym. Sci. 282(4), 330-6.

429 Zhao, C.Y., Zhang, G.H., 2011. Review on microencapsulated phase change materials (MEPCMs):

430 Fabrication, characterization and applications. Renew. Sust. Energ. Rev. 15 (8), 3813-3832. 